This item was submitted to Loughborough's Research Repository by the author.

Items in Figshare are protected by copyright, with all rights reserved, unless otherwise indicated.

\title{
Discovery of a single molecule transistor in photosystem II
}

PLEASE CITE THE PUBLISHED VERSION

http://dx.doi.org/10.1007/s10008-014-2567-z

\section{PUBLISHER}

(c) Springer-Verlag

\section{VERSION}

AM (Accepted Manuscript)

\section{PUBLISHER STATEMENT}

This work is made available according to the conditions of the Creative Commons Attribution-NonCommercialNoDerivatives 4.0 International (CC BY-NC-ND 4.0) licence. Full details of this licence are available at: https://creativecommons.org/licenses/by-nc-nd/4.0/

\section{LICENCE}

CC BY-NC-ND 4.0

\section{REPOSITORY RECORD}

Fletcher, Stephen. 2019. "Discovery of a Single Molecule Transistor in Photosystem II". figshare. https://hdl.handle.net/2134/21062. 


\title{
Discovery of a Single Molecule Transistor in Photosystem II
}

\author{
Stephen Fletcher \\ Prof S. Fletcher, Department of Chemistry, Loughborough University, Ashby \\ Road, Loughborough, Leicestershire LE11 3TU, UK. \\ E-mail: stephen.fletcher@lboro.ac.uk.
}

Submitted 21 May 2014 Accepted 30 June 2014 Published 03 August 2014

DOI: $10.1007 / s 10008-014-2567-z \quad$ C Springer-Verlag Berlin Heidelberg 2014

Keywords

Photosynthesis; Photosystem II; Transistor; Spin transition; Logic gate;

Superexchange; Electron transfer; Non-heme iron.

\begin{abstract}
Quantum theory is used to rationalize the results of recent high precision x-ray diffraction studies of photosystem II. It is proposed that a single molecule transistor regulates the flow of electrons through this remarkable system. At the core of the device, electrons flow through an iron(II) $d$-orbital by a process of superexchange, at a rate which is gated by the ambient ligand field. The transistor operates in the negative feedback mode, and its existence suggests that man-made molecular logic gates are technologically feasible. We believe this is the first recorded example of a single molecule electronic transistor in a living system.
\end{abstract}

\section{Introduction}

Photosynthesis is the conversion of carbon dioxide into biomass using energy derived from sunlight. It is an ancient biochemical process that originated shortly after life itself. Today, photosynthesis takes place inside many different organisms, including plants, algae and cyanobacteria. Indeed, among the three major domains of life (archaea, bacteria, eukarya) photosynthesis is widely distributed among the bacteria and the eukarya, although it has not been detected among the archaea [1].

Within the domain of bacteria, photosynthesis has been observed in at least five of the thirty five known phyla, namely Chlorobi (green sulfur bacteria), Chloroflexi (green non-sulfur bacteria), Cyanobacteria, Proteobacteria (purple bacteria), and Firmicutes (heliobacteria). In some cases (such as the Cyanobacteria) many species of the phylum carry out photosynthesis, whereas in other cases (such as the Proteobacteria) only a few species can perform the task.

Within the domain of eukaryotes, photosynthesis is most commonly found among the Plantae (green plants) having been imported from bacteria during the pre-Cambrian epoch [2]. Indeed, DNA sequence analysis has revealed that the chloroplasts of 
modern plants are descendants of a free-living cyanobacterium that sought refuge inside an ancient host, in an arrangement known as endosymbiosis [2-4]. Since then, the Plantae have diverged into more than 350,000 species including green algae, liverworts, mosses, ferns, and seed plants, including trees and flowering plants [5].

Due to complications caused by lateral gene transfer, much of the early evolutionary history of photosynthesis is vexed. Nevertheless, there is strong fossil evidence that photosynthetic organisms were present $\sim 3.5$ billion years ago in the form of stromatolites (microbial mats). However, since the well-attested rise in global oxygen concentration did not begin until $\sim 2.5$ billion years ago, it remains unclear whether the first oxygenic photosynthetic organisms appeared at that time, or earlier.

\section{Photosynthetic Reaction Centers}

Inside every photosynthetic organism is a special biochemical apparatus that converts light energy into electrical energy. This is known as the "reaction center". Upstream of each reaction center is an array of light-harvesting complexes that absorbs sunlight and then funnels the excitation energy towards the waiting electrons. Downstream of the reaction center are various metabolic pathways which use the excited electrons to fix carbon dioxide.

By studying the amino acid sequences of the scaffolding proteins that support different reaction centers, it has been found that reaction centers fall into two distinct categories, known as photosystem I and photosystem II. How these are distributed among living species is an interesting question. Anoxygenic organisms contain just one type of photosystem, either PSI or PSII, but never both. By contrast, oxygenic organisms always contain both types, coupled together in an arrangement known as the "Z-scheme". Thus, PSI is found in Chlorobi (green sulfur bacteria) and in Firmicutes (heliobacteria), whilst PSII is found in Proteobacteria (purple bacteria) and in Chloroflexi (green non-sulfur bacteria). Both are found in the oxygen-producing Cyanobacteria.

Despite sharing a common evolutionary origin, it is clear that PSI and PSII have diverged significantly over geological time. Today, in the PSI case, the terminal electron acceptor is an iron-sulfur complex; whereas, in the PSII case, the terminal electron acceptor is a quinone molecule. In the present study, we focus solely on PSII.

Within the PSII category, many internal differences have also developed. For example, oxygenic organisms all use water as the initial electron donor, whereas none of the anoxygenic organisms do so. Instead, they use alternative donors such as sulfur (the purple sulfur bacteria) or hydrogen (the purple non-sulfur bacteria). Yet, despite these large differences at the donor end of PSII, only small differences are observed at the acceptor end.

A schematic representation of the electron trapping states ("redox cofactors") at the acceptor end of PSII is shown in Fig. 1. It can be seen that the redox cofactors are disposed around a two-fold axis of pseudo-symmetry. Despite its near-symmetric structure, the reaction center functions asymmetrically - only the A-branch actually permits high-throughput electron tunneling. The arrows indicate the pathway of light- 
driven electron transfer. Similar one-sidedness is observed in cyanobacteria and higher plants.

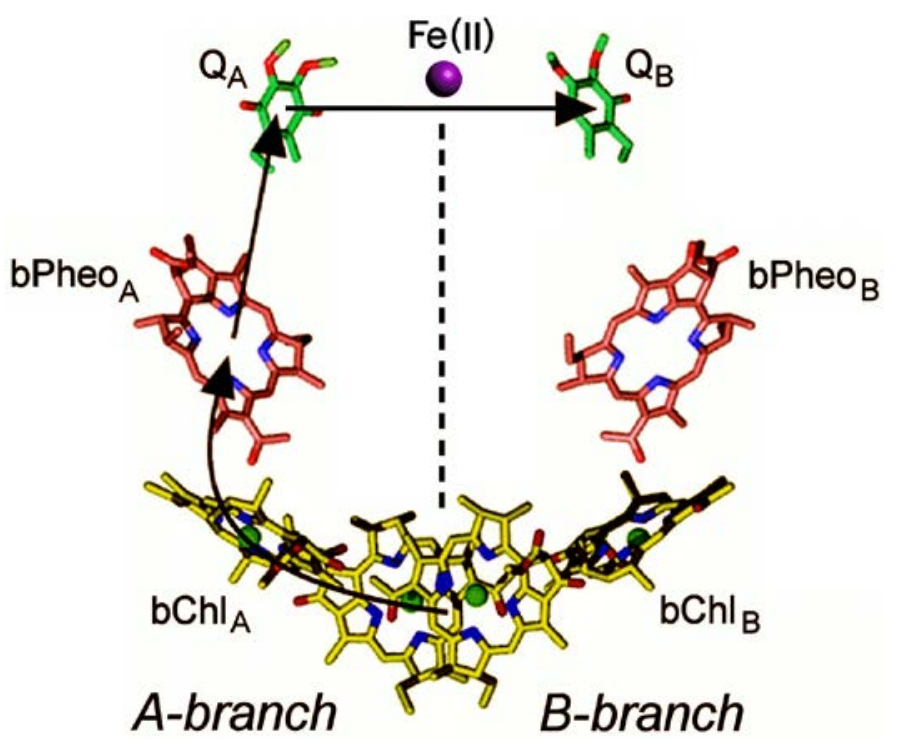

Fig. 1. Schematic representation of electron trapping states ("redox cofactors") that are necessary for the normal functioning of the bacterial reaction center of the anoxygenic purple proteobacterium Rhodobacter sphaeroides. Neighboring states have evolved within direct electron tunneling distance of each other $(<1.4 \mathrm{~nm})$. Electron transport occurs primarily through the A-branch. Supporting ligands, and a glutamate residue which provides two bonds to the non-heme iron atom, are omitted for clarity. Image derived from ref. [60].

The striking contrast between the structural symmetry and functional asymmetry in PSII has puzzled scientists for over two decades. Why has evolution retained the Bbranch if it is functionally useless? Evidently, this question can only be answered by developing a better model of electron transfer between the quinones $\mathrm{Q}_{A}$ and $\mathrm{Q}_{\mathrm{B}}$. This was one of the motivating factors behind the present work.

\section{Photosystem II}

Photosystem II (PSII) is found in the thylakoid membranes of all oxygenic photosynthetic organisms $[6,7]$. At the donor end of the chain (the lumen), sunlightinduced reactions inject electrons from water, leaving behind molecular oxygen and protons. At the acceptor end of the chain (the stroma), the emerging electrons briefly reside on a primary quinone molecule $\left(\mathrm{Q}_{\mathrm{A}}\right)$, before finally transferring to a secondary quinone molecule $\left(\mathrm{Q}_{B}\right)$. In most oxygenic organisms, such as cyanobacteria and higher plants, the secondary quinone molecule is a derivative of 2,3-dimethyl-1,4benzoquinone having an $n$-isoprenyl "tail" (a plastoquinone).

Electron transfer from the primary quinone $\mathrm{Q}_{A}$ to the secondary quinone $\mathrm{Q}_{B}$ takes place at an evolutionarily conserved reaction site known colloquially as the "nonheme iron complex". As its name implies, a distinguishing feature of this complex is that the central Fe(II) ion is not surrounded by a rigid cage of strong-field porphyrin 
ligands, unlike so many other mononuclear iron enzymes. Instead, the Fe(II) ion is surrounded by a flexible set of weak-field ligands in a distorted octahedral arrangement. As a result of this bonding motif, the $\mathrm{Fe}(\mathrm{II})$ ion is able to maintain a high spin electron configuration throughout the electron transfer reaction. Such a configuration is both surprising and puzzling. As a first-row transition element involved in an electron transfer chain, iron might be expected to exhibit multiple oxidation states during the electron transfer process. Instead, the $\mathrm{Fe}(\mathrm{II})$ ion remains stubbornly inert.

Another deeply puzzling feature of photosystem II is the mechanism by which the rate of electron flow is dynamically regulated. It has been known for over fifty years that the rate of $\mathrm{O}_{2}$ evolution depends on the intracellular concentration of $\mathrm{CO}_{2}$ (the 'bicarbonate effect'), but, so far, the mechanism of this feedback process has eluded the most rigorous enquiries [8-10].

So how does the $\mathrm{CO}_{2}$ feedback mechanism actually work? Before suggesting a solution to this historical problem, two important points should be noted. First, the direction of electron flow in photosystem II is determined by the laws of thermodynamics. In particular, the flow of electrons always occurs spontaneously from states of high Gibbs energy to states of low Gibbs energy, i.e. from states of negative redox potential to states of positive redox potential. Second, the rate of electron flow is determined by the laws of quantum mechanics, which are exquisitely sensitive to molecular position. Thus, a molecular displacement of just a few tens of picometers will cause a detectable change in the rate of electron transfer. This combination of factors suggests that the mechanism by which the rate of electron flow is dynamically regulated most probably involves a small, reversible, change in molecular position. This, in turn, suggests that we should begin our investigation by determining precisely where the key components of the electron transfer chain are actually located.

\section{The Molecular Structure of the Non-heme Iron Complex}

Over the past thirty years, the molecular structure of the photosynthetic reaction center has been one of the major targets of molecular biology. The first x-ray analysis to be reported at high resolution was that of the reaction center of the anoxygenic bacterium Rhodopseudomonas viridis by Deisenhofer et al. in 1984 [11]. This was followed a few years later by the reaction center of Rhodobacter sphaeroides, another anoxygenic species [12]. Since 2001, the molecular structure of various oxygenic photosynthetic organisms has also been elucidated at high resolution. Some of these studies are listed in Table 1.

Table 1. Some molecular structure studies of the photosynthetic reaction center since 2001.

\begin{tabular}{|l|l|l|l|l|l|}
\hline Bacterium & Resolution & Authors & Ref. & Year & Oxygenic? \\
\hline Synechococcus elongatus & $3.8 \AA$ & Zouni et al. & {$[61]$} & 2001 & yes \\
\hline Rhodobacter sphaeroides & $2.06 \AA$ & Fritzsch et al. & {$[62]$} & 2002 & no \\
\hline
\end{tabular}




\begin{tabular}{|l|l|l|l|l|l|}
\hline Rhodobacter sphaeroides & $2.35 \AA$ & Katona et al. & {$[63]$} & 2003 & no \\
\hline Thermosynechococcus vulcanus & $3.7 \AA$ & Kamiya et al. & {$[64]$} & 2003 & yes \\
\hline Thermosynechococcus elongatus & $3.5 \AA$ & Ferreira et al. & {$[65]$} & 2004 & yes \\
\hline Thermosynechococcus elongatus & $3.0 \AA$ & Loll et al. & {$[66]$} & 2005 & yes \\
\hline Thermosynechococcus elongatus & $2.9 \AA$ & Guskov et al. & {$[67]$} & 2009 & yes \\
\hline Thermosynechococcus elongatus & $3.6 \AA$ & Broser et al. & {$[68]$} & 2010 & yes \\
\hline Thermosynechococcus vulcanus & $1.9 \AA$ & Umena et al. & {$[13]$} & 2011 & yes \\
\hline
\end{tabular}

In the most recent x-ray study, Umena et al. reported the molecular structure of photosystem II from the oxygenic cyanobacterium $T$. vulcanus at a resolution of $1.9 \AA$ [13]. They found that it was composed of 17 transmembrane proteins, three peripheral proteins, and a number of cofactors, with a total molecular weight of $350 \mathrm{kDa}$. Including the antenna system, there were 35 chlorophylls, two pheophytins, $11 \beta$-carotenes, more than 20 lipids, two plastoquinones, two heme irons, four manganese atoms, three or four calcium atoms (one of which is in a $\mathrm{Mn}_{4} \mathrm{Ca}$ cluster), three chloride ions (two of which are in the vicinity of the $\mathrm{Mn}_{4} \mathrm{Ca}$ cluster), and one bicarbonate ion ligated to a non-heme iron complex. In addition, more than 1,300 water molecules were identified, the vast majority of which were external to the membrane. As predicted by ourselves a few years ago [6], only a small number of water molecules were found inside the membrane, mainly bound to chlorophyll moieties or acting as shuttles for proton transfer.

Of special interest to solid state electrochemists are the six electrochemically active cofactors that provide local minima of Gibbs energy. These cofactors are the oxygen-evolving complex (OEC), the amino acid residue tyrosine (Tyr), the reaction center chlorophyll (Chl), the molecule pheophytin (Pheo), and the plastoquinone molecules, $\mathrm{Q}_{A}$ and $\mathrm{Q}_{B}$. All of these cofactors except $\mathrm{Q}_{B}$ are bonded to a twisted pair of hydrophobic proteins known as D1 and D2. The D1 and D2 proteins form the scaffolding of the entire photosystem II complex. Each protein comprises five transmembrane helices ( $A$ to $E$ ) organized in a manner almost identical to that of the $L$ and $M$ subunits of anoxygenic photosynthetic bacteria $[14,15]$. The plastoquinone molecule $\mathrm{Q}_{B}$ is exceptional in that it is mobile. It can bind to the non-heme iron complex or it can diffuse to the quinone pool in the thylakoid membrane. When irradiated by light, electrons transfer between the cofactors, driven by the photogenerated gradient of Gibbs energy [6].

The near-symmetric spatial organization of the non-heme iron complex in photosystem II of $T$. vulcanus is illustrated in Fig. 2. These high-resolution data were obtained from the protein data bank, file number 3ARC, and ultimately derive from ref [13]. The Fe(II) ion is located approximately midway between the quinones $Q_{A}$ and $\mathrm{Q}_{\mathrm{B}}$, and is ligated by the nitrogen atoms of the imidazole moieties of four histidine (His) residues (D2-His214, D2-His268, D1-His215, D1-His272) and by a substitutionally labile bicarbonate ion (BCT-681). During photosynthesis, it is generally accepted that $\mathrm{Q}_{A}{ }^{-}$acts as a one-electron donor, while $\mathrm{Q}_{B}$ acts as a twoelectron acceptor [9]. The bicarbonate ion is bidentate, and so completes the octahedral disposition of ligands around the Fe(II) ion. In the scientific literature, it has long been recognized that the removal of bicarbonate, or its substitution by 
formate, causes a distinct slowing down of the electron transfer process by a mechanism that is, as yet, unexplained [8-10, 16].

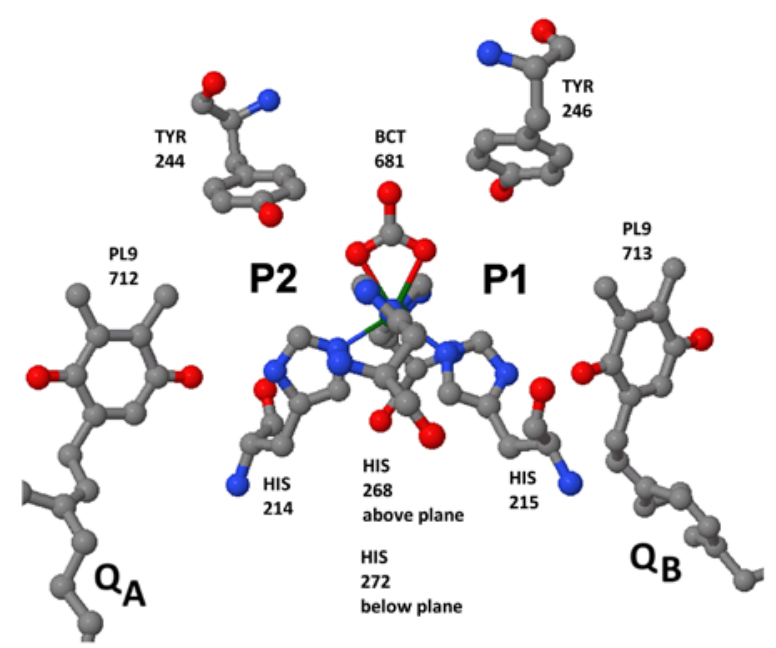

Fig. 2. The molecular structure of the non-heme iron complex of photosystem II in $T$. vulcanus, viewed in the $X Y$ plane. The Fe(II) ion is "buried" in the center of the diagram. P1 and P2 are docking pockets for the quinone head groups of $Q_{A}$ and $Q_{B}$. Structural data derived from ref [13].

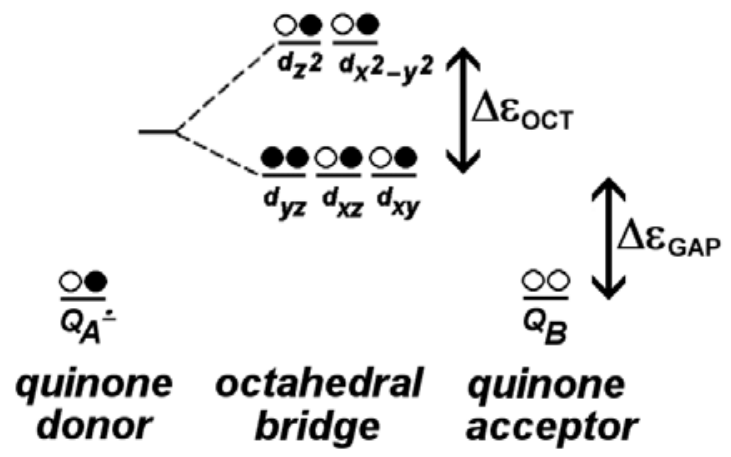

Fig. 3. Schematic molecular orbital energy diagram of the non-heme iron complex of photosystem II, in the transition state of electron transfer, neglecting minor asymmetry of the ligand field and any splitting caused by nearby semiquinone radicals. Note that four of the six $d$-electrons (black dots) in the Fe(II) center are unpaired, creating a high spin state $(S=2)$.

\section{The Electronic Structure of the Non-heme Iron Complex}

Based on the molecular structure shown in Fig. 2, we can now deduce the molecular orbital energy diagram of the non-heme iron complex in photosystem II. This is shown schematically in Fig. 3. Overall, the four histidine residues and the bicarbonate ion contribute only a small ligand field splitting energy $\Delta \varepsilon_{\text {OCT }}$ to the $d$ orbitals of Fe(II), in part due to the octahedral symmetry of the ligands around the central metal ion, and in part due to their long bond lengths. As a result of this small splitting energy, the electrons avoid pairing and distribute themselves in a high spin 
$(S=2)$ configuration. This is in full agreement with Mössbauer spectroscopy, which has clearly established that the non-heme iron complex functions in a high spin (paramagnetic) ferrous state [17]. Further evidence for the high spin $(S=2)$ configuration is provided by EPR spectroscopy [18-20] and computer simulation [21, 22]. Meanwhile, the reduced plastoquinone $\mathrm{Q}_{\mathrm{A}}{ }^{-}$has spin $1 / 2$, and its EPR spectrum has exactly the shape expected for a spin-spin interaction with a neighboring $(S=2)$ state. (As a non-Kramers ion, the high spin Fe(II) ion is itself EPR silent.) As expected, the unpaired electron on the electron donor molecule $\mathrm{Q}_{\mathrm{A}}{ }^{-}$resides in an anti-bonding $\pi_{y}$ orbital on the quinone ring [23].

For avoidance of ambiguity, we emphasize that the relative energies of the quinone orbitals shown in Fig. 3 are those of the transition state for electron transfer, not the relaxed state. In the transition state, the energies of the frontier orbitals of $\mathrm{Q}_{A}{ }^{-}$and $\mathrm{Q}_{B}$ are necessarily identical, having been temporarily equalized by thermal fluctuations in the system. By contrast, in the normal (relaxed) state, the energy of the $\mathrm{HOMO}$ of $\mathrm{Q}_{\mathrm{A}}{ }^{-}$will generally be greater than the energy of the LUMO of $\mathrm{Q}_{\mathrm{B}}$, due to the permanent gradient of electrochemical potential in the system.

The half-filled $d_{x y}$ orbital of the non-heme Fe(II) complex is of special interest in the present work because its lobes penetrate both docking pockets $\mathrm{P} 1$ and $\mathrm{P} 2$. This feature identifies the $d_{x y}$ orbital as the "missing link" between the $\mathrm{Q}_{A}{ }^{-}$and $\mathrm{Q}_{B}$ molecules. In particular, it strongly suggests that the mechanism of electron transfer is one of superexchange through the $d_{x y}$ orbital. Positive evidence in favor of this hypothesis is that the superexchange process $\pi_{y} \rightarrow d_{x y} \rightarrow \pi_{y}$ is symmetry allowed [24], so that this mode of electron transfer must occur with high probability. Negative evidence is that, in the absence of the $d_{x y}$ orbital, direct electron transfer between $\mathrm{Q}_{A}{ }^{-}$and $\mathrm{Q}_{B}$ would be practically nil, owing to the large distance between the quinone molecules.

Actually, the idea that superexchange might be implicated in electron transfer between $\mathrm{Q}_{A}{ }^{-}$and $\mathrm{Q}_{B}$ is not new, having been suggested by Fletcher in 2008 [6], and later simulated by Burggraf and Koslowski in the case of the anoxygenic species Rhodobacter sphaeroides [25]. However, in their computer simulations, these latter authors used a simplified electronic structure model which excluded the $d$-orbitals of the Fe(II) center from consideration.

As regards possible alternative pathways of electron transfer between $\mathrm{Q}_{A}{ }^{-}$and $\mathrm{Q}_{\mathrm{B}}$, we note that, on account of the Pauli Exclusion Principle, no orbital can have two electrons with the same spin, and therefore the fully-filled $d_{y z}$ orbital is spin-forbidden from taking part in electron superexchange. Similar arguments forbid electron tunneling through the four histidine residues of the protein matrix. In addition, the half-filled $d_{x z}$ orbital is out-of-plane, and therefore has the wrong spatial orientation to make a significant contribution to electron transfer along the $y$-axis. It is, however, just conceivable that hole tunneling might occur through the $d_{x 2-y 2}$ orbital (the $\mathrm{HOMO}$ ). But the HOMO is at a significantly higher energy than the LUMO due to the octahedral ligand field splitting energy, and this makes hole tunneling statistically less likely than electron tunneling. Our first major conclusion, therefore, is that the half-filled $d_{x y}$ orbital of the non-heme iron complex provides the principal superexchange channel for electron transfer between $\mathrm{Q}_{\mathrm{A}}{ }^{-}$and $\mathrm{Q}_{\mathrm{B}}$. The vast majority of electrons generated by photosystem II must surely flow through it. 
To ensure high rates of electron transfer, orbital overlap must be highly developed between the lobes of the $d_{x y}$ orbitals and the $\pi_{y}$ orbitals of $\mathrm{Q}_{A}{ }^{-}$and $\mathrm{Q}_{\mathrm{B}}$. Accordingly, we propose that the quinone molecules $\mathrm{Q}_{A}{ }^{-}$and $\mathrm{Q}_{B}$ lie deeper inside the pockets $\mathrm{P} 2$ and $\mathrm{P} 1$ in the transition state of electron transfer than they do in the relaxed state shown in Fig. 2. Remarkably, a similar conclusion was reached by Stowell et al. [26] as long ago as 1997 on purely empirical grounds. These latter authors carried out Xray diffraction experiments on reaction centers from $R$. sphaeroides in the light and in the dark, and found significant differences in the two cases. In order to distinguish them, they called the illuminated positions "proximal", and the dark positions "distal" [26].

\section{Superexchange versus Hopping}

Since the earliest days of quantum mechanics it has been known that the energy of a tunneling electron must be conserved during electron transfer between two longlived states. This conservation law compels the donor state $D$ and the acceptor state A to be of equal energy (degenerate) at the moment of electron transfer. As we have emphasized elsewhere, the necessary equalization of energies is achieved by random thermal fluctuations of electrostatic potential in the ionic atmospheres of both $D$ and $A$ [27].

In aqueous solutions, electrons cannot tunnel more than about $1.4 \mathrm{~nm}$ between free molecules [6]. However, in biological systems, it is found that electrons routinely tunnel more than $1.8 \mathrm{~nm}$. How do they do this? Careful analysis over many years has shown that the electrons do not actually tunnel directly from a donor $D$ to an acceptor A, but instead tunnel indirectly through a bridge system B, which supplies an intermediate quantum state [28-38].

Today, we know that electron transfer through intermediate quantum states may occur by two limiting mechanisms. If the energy of the intermediate state is less than $\sim 14 k_{B} T$ above the energy of the donor state (about $350 \mathrm{mV}$ at $25^{\circ} \mathrm{C}$ ), the dwell time of the electron in the intermediate state may become so long that the electron becomes bound. In this limit (known as hopping) the oxidation number of the bridge molecule temporarily decreases by one unit, and the lower oxidation state becomes detectable spectroscopically. By contrast, if the energy of the intermediate state is more than $\sim 14 k_{B} T$ above of the energy of the donor state, then the dwell time of the electron in the intermediate state is so short that the electron remains unbound. In this limit (known as superexchange) the oxidation number of the bridge molecule remains unchanged. Many authors have noted that bridge-induced superexchange can accelerate electron transfer compared with the case of no bridge at all [28-37]. And hopping can speed up electron transfer even more.

To summarize:

$\square$ "Hopping" is the tunneling of an electron between two states, mediated by one or more bound states. 
$\square \quad$ "Superexchange" is the tunneling of an electron between two states, mediated by one or more unbound states.

Given that the oxidation number of the non-heme iron complex remains unchanged during photosynthesis [38], and that the energy of the bridge state is significantly higher than that of the donor and acceptor states, we deduce that electron transfer between the quinones $\mathrm{Q}_{A}{ }^{-}$and $\mathrm{Q}_{B}$ takes place by a process of superexchange through the non-heme $\mathrm{Fe}(\mathrm{II})$ bridge system.

\section{The Rate Constant for Superexchange}

By definition, superexchange electron transfer reactions are non-adiabatic, so their rate constant is given by the "Golden Rule" expression [24]

$$
k_{\mathrm{ET}}=\frac{2 \pi}{\hbar} \int_{-\infty}^{\infty}\left\langle\left|H_{\mathrm{DBA}}(E)\right|^{2}\right\rangle \varphi_{\mathrm{DA}}(E) d E
$$

Here $k_{\mathrm{ET}}$ is the rate constant for electron transfer, $\hbar$ is the reduced Planck constant $\left(1.054 \times 10^{-34} \mathrm{~J} \mathrm{~s}\right), H_{\mathrm{DBA}}$ is the donor-bridge-acceptor coupling matrix element, $E$ is the energy of the donor state in a near-continuum of donor states of density $\varphi_{\mathrm{D}}$, and $\varphi_{\mathrm{DA}}(E)$ is the joint density of donor-acceptor states. The latter is simply the density of paired energy levels ("channels") through which superexchange can occur.

$H_{\mathrm{DBA}}$ is given by the formula

$$
H_{\mathrm{DBA}}=\frac{h_{\mathrm{DB}} h_{\mathrm{BA}}}{\Delta \varepsilon_{\mathrm{GAP}}}
$$

where $h_{\mathrm{DB}}$ and $h_{\mathrm{BA}}$ are the donor-bridge and bridge-acceptor tunneling integrals, and $\Delta \varepsilon_{\mathrm{GAP}}$ is the "energy gap" between the bridge state and the paired donor-andacceptor states (assumed degenerate) [39]. From Fig. 3, it is evident that the value of $\Delta \varepsilon_{\mathrm{GAP}}$ is strongly influenced by the octahedral ligand field splitting energy $\Delta \varepsilon_{\mathrm{OCT}}$ of the non-heme iron complex.

The theory of electron superexchange in enzymes was first developed by George and Griffith in 1959 [40], and then extended to metal ions in bridged systems by Halpern and Orgel in 1960 [41]. More recently, experimental methods have been developed which permit the measurement of the superexchange rate constant $\left(k_{\mathrm{ET}}\right)$ as a function of donor-acceptor spacing $\left(R_{\mathrm{DA}}\right)$. In many cases the experimental data have been arbitrarily fitted to an equation of the form

$$
k_{\mathrm{ET}}=\alpha \exp \left(-\beta R_{\mathrm{DA}}\right)
$$

where $\alpha$ is a pre-exponential factor and $\beta$ is a decay constant characteristic of the bridge and the DA pair. Typically, DBA systems having conjugated bridges show low 
$\beta$ values $\left(<4 \mathrm{~nm}^{-1}\right)$, while DBA systems having unconjugated (alkane) bridges show slightly higher $\beta$ values $\left(>8 \mathrm{~nm}^{-1}\right.$ ) [34, 42-44]. In all cases, however, it has been observed that the rate constants for superexchange are very high when the donoracceptor spacing is small.

\section{Ligand Field Modulation of Superexchange}

In addition to the donor-acceptor spacing $\left(R_{\mathrm{DA}}\right)$, the energy gap $\Delta \varepsilon_{\mathrm{GAP}}$ also has a very powerful influence on the rate constant for superexchange, via Eq. (2). In this context, the recent $1.9 \AA$ crystal structure of photosystem II is particularly important, because it has revealed that a bicarbonate ion is bound to the non-heme iron complex [13]. As noted earlier, the bicarbonate ion is bidentate, and therefore is able to complete the octahedral arrangement of ligands around the Fe(II) center. The resulting molecular orbital energy diagram was sketched in Fig. 2. We now ask the question, "What would happen if the bidentate ligand were to be replaced by a monodentate ligand, such as cyanide?"

Clearly, the system would be forced into five-coordinate geometry, essentially a square pyramid with the four histidine ligands at the corners of the base and the cyanide ion at the apex. If we allow for a 90 degree rotation of the co-ordinate system (a Berry-type rotation, so that the $x-y$ plane is now taken as the base of the square pyramid rather than the equator of the octahedron) then the changes in the molecular orbital energy diagram may be simply summarized as: (i) a lowering of the energies of the $d_{y z}$ and $d_{x z}$ orbitals, and (ii) a raising of the energy of the $d_{x 2-y 2}$ orbital. Although these changes are seemingly minor, they actually have some very major consequences. Most important of all, the ligand field splitting energy now exceeds the electron spin-pairing energy, causing the low-lying orbitals to fill up with paired electrons (Fig. 4).

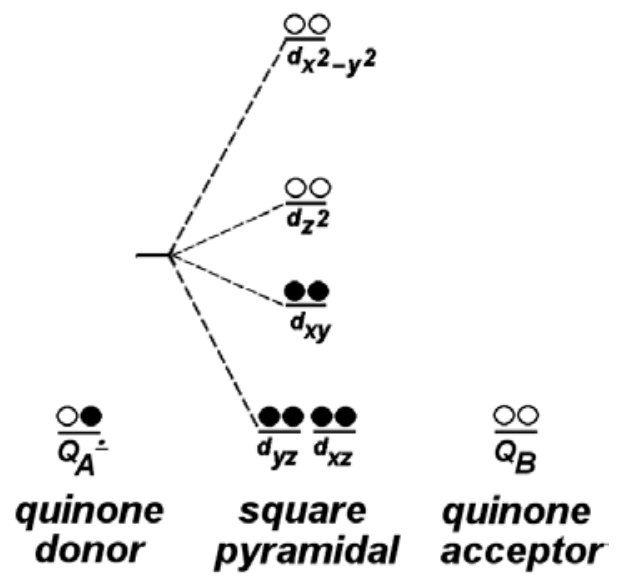

Fig. 4. Schematic molecular orbital energy diagram of the non-heme iron complex after a weak-field ligand (bicarbonate) has been substituted by a strong-field ligand (cyanide). All six of the $d$-electrons in the Fe(II) center have now paired up, causing a spin transition from a paramagnetic $(S=2)$ state to a diamagnetic $(S=0)$ state. Due to the complete filling of the low-lying $d$-orbitals, the electron superexchange process between $\mathrm{Q}_{A}{ }^{-}$and $\mathrm{Q}_{B}$ is blocked. 
Accordingly, the non-heme iron complex undergoes a spin transition from a paramagnetic $(S=2)$ state to a diamagnetic $(S=0)$ state, and the superexchange channel between $\mathrm{Q}_{A}{ }^{-}$and $\mathrm{Q}_{B}$ becomes blocked by Pauli exclusion. These outcomes correspond precisely to experimental observations. Accordingly, our second major conclusion is that the field strength of the incoming ligand controls the rate of superexchange of electrons between $\mathrm{Q}_{A}{ }^{-}$and $\mathrm{Q}_{B}$, with the maximum rate occurring in the case of a weak field, and the minimum rate in the case of a strong field.

We can now make some useful generalizations, and some important predictions, about the behavior of photosystem II. For example, our model suggests that all monodentate ligands which are ranked above medium strength in the spectrochemical series (see Table 2) should have an inhibiting effect on electron transfer in photosystem II, provided they are present in sufficient concentration to displace bicarbonate ions. Interestingly, the extensive literature on the "bicarbonate effect" already provides some tentative support for this view in the case of azide, fluoride, nitrate, and nitrite ions [45-51]. Strong evidence of the potent inhibitory effect of cyanide ions also exists. For example, by using Mössbauer spectroscopy, Sanakis et al. [46] found that cyanide ions convert non-heme iron complexes from the high spin state $(S=2)$ to the low spin state $(S=0)$, just as the present theory says they should. In addition, the biochemical reversibility of the bicarbonate effect — also implicit in the theory — was demonstrated long ago by Govindjee et al. [49]. Finally, Hermes et al. [38] have performed time-resolved x-ray absorption measurements which confirm that the $\mathrm{Fe}(\mathrm{II})$ ion does not change oxidation state during the $\mathrm{Q}_{\mathrm{A}}{ }^{-} \rightarrow \mathrm{Q}_{\mathrm{B}}$ electron transfer process, thus providing powerful support to the superexchange interpretation.

Table 2. Spectrochemical series of selected strong-field ligands in $d^{6}$ complexes of transition metal ions. The precise values of the ligand field splitting energies for $\mathrm{Fe}(\mathrm{II})$ complexes are not known, but they are thought to be within 5\% of the values for $\mathrm{Co}(\mathrm{III})$ complexes listed below [69]. The ligand field splitting energies $(\Delta)$ are given in units of eV per ligand.

\begin{tabular}{|l|l|l|l|l|l|}
\hline $\mathrm{N}_{3}^{-}$ & $\mathrm{F}^{-}$ & $\mathrm{NO}_{3}^{-}$ & $\mathrm{OH}^{-}$ & $\mathrm{NO}_{2}^{-}$ & $\mathrm{CN}^{-}$ \\
\hline 0.287 & 0.307 & 0.313 & 0.318 & 0.523 & 0.633 \\
\hline
\end{tabular}

Given that the non-heme iron complex is able to change its spin state in response to the replacement of a single ligand, it follows immediately that the total ligand field strength inside the complex is delicately poised to allow this. This is a likely reason why the structure of the complex has been so resistant to evolutionary change.

The development of ideas about the bicarbonate-reversible catalysis of photosynthesis has a long and fascinating history. The modern epoch began in 1975, when Wydrzynski and Govindjee demonstrated that depletion of bicarbonate slowed down the rate of electron transfer on the electron acceptor side of PSII [47]. By 1984, Eaton-Rye and Govindjee [48] had narrowed down the site of the inhibition to the $\mathrm{Q}_{A}{ }^{-} / \mathrm{Q}_{B}$ reaction, and later that year Govindjee et al. [49] found that the effect 
could be reversed by adding fresh bicarbonate ions to previously-depleted samples. Also in 1984, Snel and Van Rensen [50] described the inhibiting effects of formate ions, which are widely-distributed by-products of photorespiration. In 1985, Stemler and Murphy reported the bicarbonate-reversible inhibition of photosystem II by fluoride, nitrite, nitrate, acetate and formate ions [51], and finally in 1994 Sanakis et al. [46] showed the dramatic effects of cyanide ions.

By the mid-nineties, the modern consensus had finally been reached, namely that (1) a wide diversity of singly-charged anions can inhibit oxygenic photosynthesis, (2) reactivation is unique to the bicarbonate ion, and (3) the effect is absent in anoxygenic organisms [50]. In the two decades since, a deeper understanding of these facts has proved elusive. Now, however, with the publication of the molecular structure of photosystem II from $T$. vulcanus at $1.9 \AA$ resolution [13], we have sufficient information to explain what is happening. The key factor is that the bicarbonate ion creates a weak ligand field, whereas all the inhibiting anions create a strong ligand field. This causes spin-pairing, which in turn blocks the superexchange process. By contrast, in anoxygenic species, the bicarbonate ion is permanently replaced by a bidentate glutamate ligand (M234 in Rhodobacter sphaeroides, M232 in $R$ viridis) and substitution by monodentate ions is prevented. (It should also be noted that some as-yet undiscovered conformational control of the rate of electron superexchange may exist in anoxygenic species, operating through the mechanical position of the glutamate ligand, but this remains a speculative proposition for the moment.)

Macroscopically, oxygen and carbon dioxide mutually displace each other from water. Therefore, high oxygen concentration means low carbon dioxide concentration, and vice versa. Further, in aqueous solution, the bicarbonate ion is in equilibrium with molecular carbon dioxide via the dissociation of carbonic acid:

$\mathrm{CO}_{2}+\mathrm{H}_{2} \mathrm{O}=\mathrm{H}_{2} \mathrm{CO}_{3}$

$\mathrm{H}_{2} \mathrm{CO}_{3}=\mathrm{HCO}_{3}^{-}+\mathrm{H}^{+}$

So, by coupling the bicarbonate ion concentration to the rate of electron superexchange, oxygenic photosynthetic organisms are able to implement a form of negative feedback, such that the rate of electron transfer is low when the oxygen concentration is high.

\section{A Transistor Appears}

Viewed from the standpoint of electrical engineering, it is apparent that the nonheme iron complex in photosystem II is behaving as a single molecule transistor in the negative feedback mode. The analogy with a junction gate field-effect transistor (JFET) is very close, as shown in Fig. 5. The JFET is a multi-molecule transistor that uses an electric field to control the flow of electrons through a macroscopic semiconduction channel. The non-heme iron complex is a single molecule transistor that uses a ligand field to control the flow of electrons through a nanoscopic superexchange channel. This astonishing parallelism suggests the possibility of 
constructing a whole new generation of single molecule electronic devices, based on the ligand field modulation of the superexchange process.

The use of transistors for the construction of logic gates depends upon their ability to perform as switches. The existence of a single molecule transistor in nature that behaves as a switch at room temperature is therefore compelling evidence that single molecule logic gates are technologically feasible. Indeed, this discovery raises the tantalizing possibility that the whole transistor-transistor-logic (TTL) family might one day be re-created on a molecular scale.
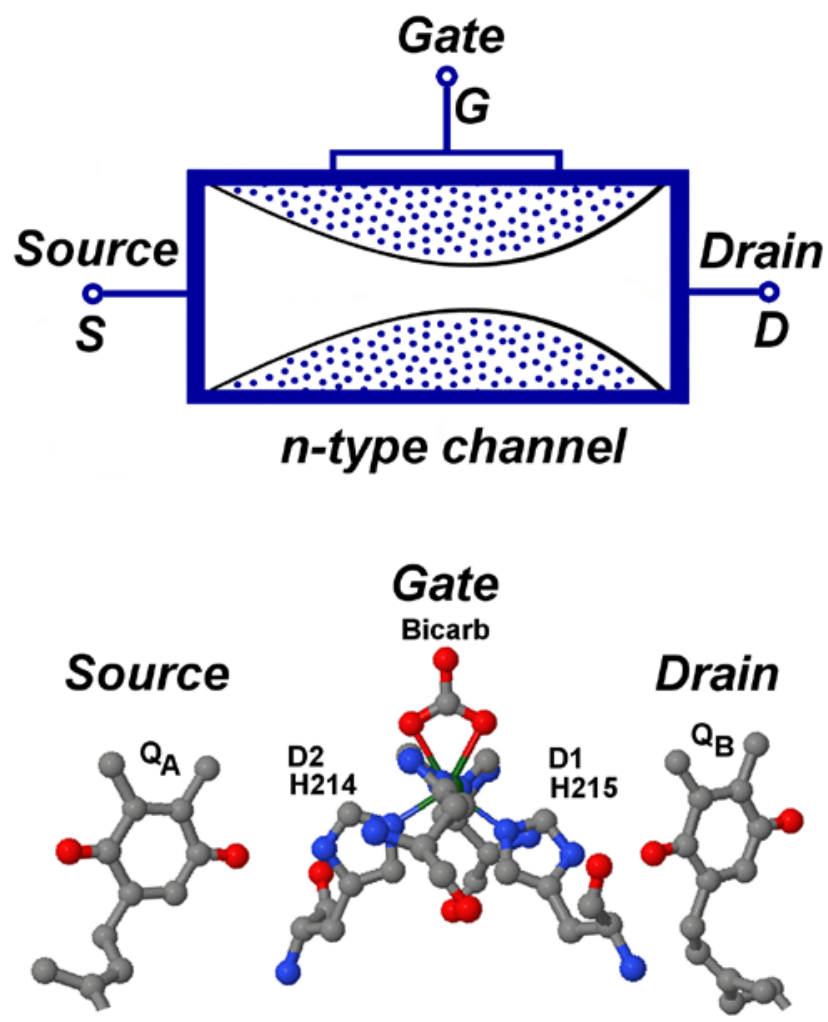

Fig. 5. Comparison of a JFET with the single molecule transistor in PSII. Both are three-terminal devices, consisting of a source, a gate, and a drain. Top: The JFET uses an electric field to "gate" the conduction of electrons between source and drain. Bottom: The molecular transistor uses the bicarbonate ligand field to "gate" the superexchange of electrons between $\mathrm{Q}_{\mathrm{A}}$ and $\mathrm{Q}_{\mathrm{B}}$.

\section{Conclusions}

In a recent x-ray study, Umena et al. determined the molecular structure of photosystem II from the oxygenic cyanobacterium $T$. vulcanus at a resolution of $1.9 \AA$ [13]. They found that the central Fe(II) ion in the non-heme iron complex was bonded to four monodentate histidine residues and to one bidentate bicarbonate ligand, arranged in a distorted octahedron. In addition, the entire complex was located midway between the primary electron donor $\mathrm{Q}_{A}{ }^{-}$and the secondary electron acceptor $\mathrm{Q}_{\mathrm{B}}$ of the electron transfer chain. In the present work, we have analyzed this unusual structure from a quantum mechanical perspective, and have discovered 
that the non-heme iron complex is behaving as a single molecule transistor that regulates the flow of electrons between $\mathrm{Q}_{A}$ and $\mathrm{Q}_{B}$.

The role of the non-heme iron complex in photosynthesis has puzzled scientists for a long time, largely because the Fe(II) ion does not change oxidation state during electron transfer. Why is this? In the present work, we have suggested that the answer lies in the very short residence time of the mobile electron in the $\mathrm{Fe}$ (II) center as it superexchanges between the quinones $\mathrm{Q}_{\mathrm{A}}$ and $\mathrm{Q}_{\mathrm{B}}$.

Another puzzle of long standing is the regulatory role of the bicarbonate ion in photosystem II. As noted earlier in this text, the rate of oxygen evolution depends on the intracellular concentration of bicarbonate ions, but, so far, the mechanism of this feedback process has remained unsolved [8-10]. Here, we have suggested that the key factor is the weak ligand field strength of the bicarbonate ion as it bonds with the non-heme iron complex. A weak ligand field prevents the spin-pairing of the frontier electrons. As a result, the superexchange orbital remains open for electron transfer, and photosynthesis occurs. By contrast, a strong ligand field created by ions such as cyanide induces spin-pairing of the frontier electrons. This blocks the superexchange orbital, and photosynthesis switches off (Fig. 6).

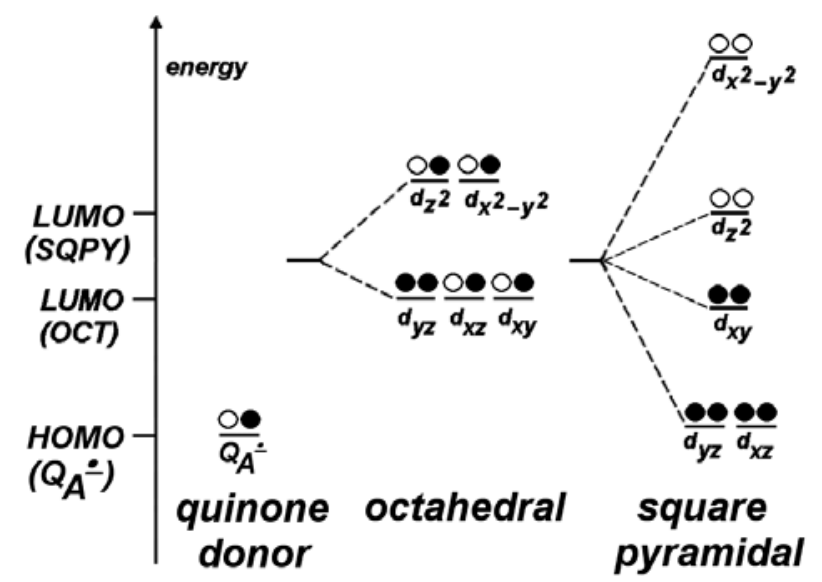

Fig. 6. Effect of the Octahedral-to-Square-Pyramidal transition on the molecular orbital energy diagram of the non-heme iron complex (schematic). Depletion of electron charge in the upper (anti-bonding) orbitals shortens the metal-to-ligand bond distances and thereby increases the ligand field splitting energy.

The precise location of the bicarbonate ion in the spectrochemical series is not known, but if we assume that it is a weak $\pi$-donor then it would have $\Delta \sim 0.260$ $\mathrm{eV} /$ ligand. If this is correct, then the minimum thermal energy required to switch electron transfer on and off would be of the order of a few $k_{B} T$. Interestingly, such a small activation energy would imply the existence of a second type of spin transition in addition to the ligand-induced spin transition discussed above. This would be a thermally-induced spin transition at cryogenic temperatures. If such a transition could be found in the non-heme iron complex then that would provide a very powerful endorsement of the present model, particularly when it is considered that no chemical reaction would be involved. 
How might one locate such a transition? Well, examination of the literature on the very few iron(II) $d^{6}$ complexes that are already known to display thermally-induced spin transitions $[52,53]$ suggests that a transition from a high spin to a low spin state would most likely occur in the temperature range $170-230 \mathrm{~K}$. If that were the case in photosystem II, then we should expect the electron transfer between $\mathrm{Q}_{A}{ }^{-}$and $\mathrm{Q}_{B}$ to switch off in the same temperature range. This is an unambiguous prediction that could be used as a critical test of our model.

Historically, there have been several previous claims of transistor-like behavior in biological systems. For example, voltage-gated ion channels that control electrical activity in nerve, muscle and many other cell types have been referred to as "transistors" or "transistor-like" in their action [54,55]. However, the charge carriers in those cases were not electrons but ions. Similarly, transistor-like behavior has been attributed to "genetic logic gates" [56], but these use double-stranded DNA molecules to reproduce base pair sequences, not electrons. To date, therefore, there appears to be no example of a "genuine" bio-transistor operating on electron flow. Accordingly, we believe that the non-heme iron complex analyzed in the present work is the first recorded example of such a device.

Finally, the results of the present work provide fresh insights into some unsolved problems of environmental science and crop productivity. Here, we note just three. First, the model explains in detail why carbon dioxide stimulates plant growth inside greenhouses [57], and also explains why the beneficial effect saturates at high concentration (the bicarbonate ions simply fill all the available ligand sites in the nonheme iron complexes). Second, the model explains why concentrated nitrate fertilizer may be safely applied to plant roots but not to plant leaves (on leaves, the nitrate ions block photosynthesis, leading to "leaf burn" [58]). Third, the model explains why large-scale cyanide fishing "bleaches" coral reefs in many tropical regions of the world [59] (the cyanide ions powerfully inhibit photosynthesis in the symbiotic algae [species zooxanthellae] that are needed for coral production). Doubtless many further insights await discovery.

\section{Acknowledgements}

I thank Prof Vickie McKee (Loughborough) for assistance with computer visualization of coordination complexes of Fe(II), and Prof. Dr. Winfried Hinrichs (Greifswald) for helpful comments on the manuscript.

\section{References}

[1] Bryant DA, Frigaard N-U (2006) Prokaryotic photosynthesis and phototrophy illuminated. Trends in Microbiology 14:488-496

[2] Sagan, L (1967) On the origin of mitosing cells. Journal of Theoretical Biology $14: 255-274$

[3] Curtis SE, Clegg MT (1984) Molecular evolution of chloroplast DNA sequences. Molecular Biology and Evolution 1:291-301 
[4] Deusch O, Landan G, Roettger M, Gruenheit N, Kowallik KV, Allen JF, Martin W, Dagan T (2008) Genes of cyanobacterial origin in plant nuclear genomes point to a heterocyst-forming plastid ancestor. Molecular Biology and Evolution 25:748-761

[5] Smith SA, Beaulieu JM, Donoghue MJ (2009) Mega-phylogeny approach for comparative biology: an alternative to supertree and supermatrix approaches. BMC Evolutionary Biology 9:37 (12 pp)

[6] Fletcher S (2008) The new theory of electron transfer: application to the photosynthetic reaction centre. Journal of Solid State Electrochemistry 12:1511-1520

[7] Fletcher S (2011) Electronomics. Journal of Solid State Electrochemistry 15:1451-1458

[8] Warburg O, Krippahl G (1958) Hill-Reaktionen. Z. Naturforsch B 13:509-514

[9] Shevela D, Eaton-Rye JJ, Shen JR, Govindjee (2012) Photosystem I/ and the unique role of bicarbonate: a historical perspective. Biochim Biophys Acta 1817:1134-51

[10] Carrieri D, Ananyev G, Brown T, Dismukes GC (2007) In vivo bicarbonate requirement for water oxidation by photosystem II in the hypercarbonate-requiring cyanobacterium Arthrospira maxima. J Inorg Biochem 101:1865-74

[11] Deisenhofer J, Epp O, Miki K, Huber R, Michel H (1984) X-ray structure analysis of a membrane protein complex. Electron density map at $3 \AA$ resolution and a model of the chromophores of the photosynthetic reaction center from Rhodopseudomonas viridis. J Mol Biol 180:385-398

[12] Allen JP, Feher G, Yeates TO, Komiya H, Rees DC (1987) Structure of the reaction center from Rhodobacter sphaeroides $R-26$ : the cofactors. Proc Natl Acad Sci USA 84:5730-5734

[13] Umena Y, Kawakami K, Shen J-R, Kamiya N (2011) Crystal structure of oxygenevolving photosystem II at a resolution of $1.9 \AA$. Nature 473:55-60

[14] Allen JP, Feher G, Yeates TO, Komiya H, Rees DC (1988) Structure of the reaction center from Rhodobacter sphaeroides $R-26$ : protein-cofactor (quinones and Fe2+) interactions. Proc Natl Acad Sci USA 85:8487

[15] Deisenhofer J, Michel H (1989) Nobel lecture. The photosynthetic reaction centre from the purple bacterium Rhodopseudomonas viridis. EMBO J 8:2149-2170

[16] Petrouleas V, Crofts AR (2005) The iron-quinone acceptor complex. Photosystem II: The light-driven water:plastoquinone oxidoreductase. Eds. Wydrzynski T, Satoh K (Springer, Dordrecht, The Netherlands), pp 177-206

[17] Hałas A, Orzechowska A, Derrien V, Chumakov Al, Sebban P, Fiedor J, Lipińska M, Zając M, Ślęzak T, Strzałka K, Matlak K, Korecki J, Fiedor L, Burda K (2012) The dynamics of the non-heme iron in bacterial reaction centers from Rhodobacter sphaeroides. Biochim Biophys Acta 1817:2095-2102 
[18] Nugent JHA, Diner BA, Evans MCW (1981) Direct detection of the electron acceptor of photosystem II: Evidence that $Q$ is an iron-quinone complex. FEBS Letters 124:241-244

[19] Rutherford AW, Zimmermann J-L (1984) A new EPR signal attributed to the primary plastosemiquinone acceptor in Photosystem II. Biochimica et Biophysica Acta-Bioenergetics 767:168-175

[20] Nugent JHA, Doetschman DC, MacLachlan DJ (1992) Characterization of the multiple EPR line shapes of iron-semiquinones in photosystem II. Biochemistry 31:2935-2941

[21] Butler WF, Calvo R, Fredkin DR, Isaacson RA, Okamura MY, and Feher G (1984) The electronic structure of Fe2+ in reaction centers from Rhodopseudomonas sphaeroides. III. EPR measurements of the reduced acceptor complex. Biophysical journal 45:947-973

[22] Dismukes GC, Frank HA, Friesner R, Sauer K (1984) Electronic interactions between iron and the bound semiquinones in bacterial photosynthesis. EPR spectroscopy of oriented cells of Rhodopseudomonas viridis. Biochimica et Biophysica Acta (BBA)-Bioenergetics 764:253-271

[23] O'Malley PJ (1998) B3LYP hybrid density functional studies of the durosemiquinone radical: The effect of symmetrical and asymmetrical hydrogen bonding on spin densities and hyperfine couplings. J Phys Chem A 102:248-253

[24] Fletcher S (2010) The theory of electron transfer. Journal of Solid State Electrochemistry 14:705-739

[25] Burggraf F, Koslowski T (2011) The simulation of interquinone charge transfer in a bacterial photoreaction center highlights the central role of a hydrogen-bonded non-heme iron complex. Biochimica et Biophysica Acta 1807:53-58

[26] Stowell MHB, McPhillips TM, Rees DC, Soltis SM, Abresch E, Feher G (1997) Light-induced structural changes in photosynthetic reaction center: implications for mechanism of electron-proton transfer. Science, 276:812-816.

[27] Fletcher S (2007) A non-Marcus model for electrostatic fluctuations in long range electron transfer. Journal of Solid State Electrochemistry 11:965-969

[28] Hopfield JJ (1974) Electron transfer between biological molecules by thermally activated tunneling. Proc Natl Acad Sci USA 71:3640-3644

[29] Jortner J (1980) Dynamics of electron transfer in bacterial photosynthesis. Biochim Biophys Acta 594:193-230

[30] Marcus RA, Sutin N (1985) Electron transfers in chemistry and biology. Biochim Biophys Acta 811:265-322

[31] Sigel H, Sigel A (1991) Electron Transfer Reactions in Metalloproteins. Eds. Sigel H, Sigel A (Marcel Dekker, New York, NY) 
[32] Gray HB, Winkler JR (1996) Electron transfer in proteins. Annu. Rev. Biochem. 65:537-561

[33] Regan JJ, Ramirez BE, Winkler JR, Gray HB, Malmstrom BG (1998) Pathways for electron tunneling in cytochrome $c$ oxidase. J Bioenerg Biomembr 30:35-39

[34] Page CC, Moser CC, Chen X, Dutton PL (1999) Natural engineering principles of electron tunneling in biological oxidation reduction. Nature 402:47-52

[35] Regan JJ, Onuchic JN (1999). Electron-transfer tubes. Adv Chem Phys 107:497-553

[36] Segal D, Nitzan A, Davis WB, Wasielewski MR, Ratner MA (2000) Electron transfer rates in bridged molecular systems 2. A steady-state analysis of coherent tunneling and thermal transitions. J Phys Chem B 104:3817-3829

[37] Saito K, Kikuchi T, Mukai K, Sumi H (2009) Sequential or superexchange mechanism in bridged electron transfer distinguished by dynamics at a bridging molecule. Phys Chem Chem Phys 11:5290-5301

[38] Hermes S, Bremm O, Garczarek F, Derrien V, Liebisch P, Loja P, Sebban P, Gerwert K, Haumann M (2006) A time-resolved iron-specific x-ray absorption experiment yields no evidence for an $\mathrm{Fe}^{2+} \rightarrow \mathrm{Fe}^{3+}$ transition during $\mathrm{Q}_{A}{ }^{-} \rightarrow \mathrm{Q}_{B}$ electron transfer in the photosynthetic reaction center. Biochemistry 45:353-3540

[39] McConnell HM (1961) Intramolecular charge transfer in aromatic free radicals. The Journal of Chemical Physics 35:508-515

[40] George P, Griffith JS (1959) Electron transfer and enzyme catalysis. Chapter 8 in "The Enzymes", Eds. Boyer PD, Lardy H, Myrback K (Academic Press, NY, pp 347-389)

[41] Halpern J, Orgel LE (1960) The theory of electron transfer between metal ions in bridged systems. Discussions of the Faraday Society 29:32-41

[42] Moser CC, Keske JM, Warncke K, Farid RS, Dutton PL (1992) Nature of biological electron transfer. Nature 355:796-802

[43] Lewis FD, Liu J, Weigel W, Rettig W, Kurnikov IV, Beratan DN (2002) Donorbridge-acceptor energetics determine the distance dependence of electron tunneling in DNA. Proceedings of the National Academy of Sciences 99:12536-12541

[44] Moser CC, Anderson JL, Dutton PL (2010) Guidelines for tunneling in enzymes. Biochimica et Biophysica Acta (BBA)-Bioenergetics, 1797:1573-1586

[45] Govindjee (1993) Bicarbonate-reversible inhibition of plastoquinone reductase in photosystem II, Z. Naturforsch. C, 48:251-258 
[46] Sanakis Y, Petrouleas V, Diner BA (1994) Cyanide binding at the non-heme Fe2+ of the iron-quinone complex of photosystem II: At high concentrations, cyanide converts the Fe2+ from high $(S=2)$ to low $(S=0)$ spin. Biochemistry, 33:9922-9928

[47] Wydrzynski T, Govindjee (1975) New site of bicarbonate effect in photosystem II of photosynthesis - evidence from chlorophyll fluorescence transients in spinach chloroplasts. Biochim Biophys Acta 387:403-408

[48] Eaton-Rye JJ, Govindjee (1984) A study of the specific effect of bicarbonate on photosynthetic electron transport in the presence of methyl viologen. Photobiochem Photobiophys 8:279-288

[49] Govindjee, Nakatani HY, Rutherford AW, Inoue Y (1984) Evidence from thermoluminescence for bicarbonate action on the recombination reactions involving the secondary quinone electron acceptor of photosystem II. Biochim Biophys Acta $766: 416-423$

[50] Snel JF, van Rensen JJ (1984) Reevaluation of the role of bicarbonate and formate in the regulation of photosynthetic electron flow in broken chloroplasts. Plant Physiology 75:146-150

[51] Stemler A, Murphy JB (1985) Bicarbonate-reversible and irreversible inhibition of photosystem II by Monovalent Anions. Plant Physiology 77:974-977

[52] Baker WA, Bobonich HM (1964) Magnetic Properties of Some High-Spin Complexes of Iron(II). Inorg Chem 3:1184-1188

[53] Gütlich P, Hauser A, Spiering H (1994) Thermal and optical switching of iron (II) complexes. Angewandte Chemie International Edition in English 33:2024-2054

[54] Sigworth FJ (2003) Life's transistors. Nature 423:21-22

[55] Jiang Y, Lee A, Chen J, Ruta V, Cadene M, Chait BT, MacKinnon R (2003) Xray structure of a voltage-dependent $K+$ channel. Nature 423:33-41

[56] Bonnet J, Yin P, Ortiz ME, Subsoontorn P, Endy D (2013) Amplifying Genetic Logic Gates. Science 340:599-603

[57] Kirkham MB (2013) Research Needs for Agriculture under Elevated Carbon Dioxide. Chapter 12 in Handbook of Climate Change and Agroecosystems: Global and Regional Aspects and Implications. Eds. Hillel D, Rosenzweig C (Imperial College Press, London) 225-234

[58] Walsh OS, Christiaens RJ, Pandey A (2013). US West: Foliar-applied nitrogen fertilizers in spring wheat production. Crops and Soils 46:26-32

[59] Jones RJ, Hoegh-Guldberg O (1999) Effects of cyanide on coral photosynthesis: implications for identifying the cause of coral bleaching and for assessing the environmental effects of cyanide fishing. Marine Ecology Progress Series 177:83-91 
[60] Wakeham MC, Jones MR (2005) Rewiring photosynthesis: engineering wrongway electron transfer in the purple bacterial reaction centre. Biochemical Society Transactions 33:851-857

[61] Zouni A, Witt HT, Kern J, Fromme P, Krauss N, Saenger W, Orth P (2001) Crystal structure of photosystem II from Synechococcus elongatus at $3.8 \AA$ resolution. Nature 409:739-743

[62] Fritzsch G, Koepke J, Diem R, Kuglstatter A, Baciou L (2002) Charge separation induces conformational changes in the photosynthetic reaction centre of purple bacteria. Acta Crystallog Sect. D 58:1660-1663

[63] Katona G, Andréasson U, Landau EM, Andréasson L-E, Neutze R (2003) Lipidic cubic phase crystal structure of the photosynthetic reaction centre from Rhodobacter sphaeroides at 2.35 A Resolution. Journal of Molecular Biology 331:681-692

[64] Kamiya N, Shen J-R (2003) Crystal structure of oxygen-evolving photosystem II from Thermosynechococcus vulcanus at 3.7 A resolution. Proc Natl Acad Sci USA 100:98

[65] Ferreira KN, Iverson TM, Maghlaoui K, Barber J, Iwata S (2004) Architecture of the photosynthetic oxygen-evolving center. Science 303:1831-1838

[66] Loll B, Kern J, Saenger W, Zouni A, Biesiadka J (2005) Towards complete cofactor arrangement in the $3.0 \AA$ resolution structure of photosystem II. Nature 438:1040-1044

[67] Guskov A, Kern J, Gabdulkhakov A, Broser M, Zouni A, Saenger W, Cyanobacterial photosystem II at 2.9 A resolution and the role of quinones, lipids, channels and chloride. Nature Structural and Molecular Biology 16:334-342

[68] Broser M, Gabdulkhakov A, Kern J, Guskov A, Müh F, Saenger W, Zouni A (2010) Crystal structure of monomeric photosystem II from Thermosynechococcus elongatus at $3.6 \AA$ resolution. The Journal of Biological Chemistry, 285:26255-26262 [69] Shimura Y (1988) A quantitative scale of the spectrochemical series for the mixed ligand complexes of $d 6$ metals. Bulletin of the Chemical Society of Japan 61:693-698 